\title{
Pre-incubation with hucMSC-exosomes prevents cisplatin-induced nephrotoxicity by activating autophagy
}

Bingying Wang ${ }^{1,2 \dagger}$, Haoyuan Jia ${ }^{1 \dagger}$, Bin Zhang ${ }^{1}$, Juanjuan Wang ${ }^{1}$, Cheng $\mathrm{Ji}^{1}$, Xueming Zhu ${ }^{2}$, Yongmin Yan ${ }^{1}$, Lei Yin ${ }^{1}$, Jing $\mathrm{Yu}^{1}$, Hui Qian ${ }^{1 *}$ (D) and Wenrong $X \mathrm{u}^{{ }^{*}}$

\begin{abstract}
Background: The administration of cisplatin is limited due to its nephrotoxic side effects, and prevention of this nephrotoxicity of cisplatin is difficult. Mesenchymal stem cell (MSC)-derived exosomes have been implicated as a novel therapeutic approach for tissue injury. In this study, we demonstrated that the pretreatment of human umbilical cord MSC-derived exosomes (hucMSC-Ex) can prevent the development of cisplatin-induced renal toxicity by activation of autophagy in vitro and in vivo.

Methods: In vitro, rat renal tubular epithelial (NRK-52E) cells were pre-incubated with exosomes from hucMSC or HFL1 (human lung fibroblast cells; as control) for $30 \mathrm{~min}$, and 3-methyladenine (an autophagic inhibitor) and rapamycin (an autophagic inducer) for $1 \mathrm{~h}$ before cisplatin treatment for $8 \mathrm{~h}$, respectively. Cells were harvested for apoptosis assay, enzyme-linked immunosorbent assay (ELISA), Western blot, and quantitative real-time polymerase chain reaction (qRT-PCR). In vivo, we constructed cisplatin-induced acute kidney injury rat models. Prior to treatment with cisplatin for 0.5 h, huCMSC-Ex or HFL1-Ex were injected into the kidneys via the renal capsule. 3-methyladenine and rapamycin were injected under the kidney capsule before hucMSC-Ex. All animals were sacrificed at 3 days after cisplatin injection. Renal function, Luminex assay, tubular apoptosis and proliferation, and autophagy response were evaluated.
\end{abstract}

Results: hucMSC-Ex inhibited cisplatin-induced mitochondrial apoptosis and secretion of inflammatory cytokines in renal tubular epithelial cells in vitro. hucMSC-Ex increased the expression of the autophagic marker protein LC3B and the autophagy-related genes ATG5 and ATG7 in NRK-52E cells. Rapamycin mimicked the effects of hucMSC-Ex in protecting against cisplatin-induced renal injury, while the effects were abrogated by the autophagy inhibitor 3-methyladenine in the animals.

Conclusions: Our findings indicate that the activation of autophagy induced by hucMSC-Ex can effectively relieve the nephrotoxicity of cisplatin. Therefore, pre-treatment of hucMSC-Ex may be a new method to improve the therapeutic effect of cisplatin.

Keywords: Human umbilical cord mesenchymal stem cell, Exosome, Cisplatin, Nephrotoxicity, Autophagy

\footnotetext{
* Correspondence: Istmmmlst@163.com; icls@ujs.edu.cn

†Equal contributors

${ }^{1}$ Key Laboratory of Laboratory Medicine of Jiangsu Province, School of

Medicine, Jiangsu University, 301 Xuefu Road, Zhenjiang, Jiangsu 212013,

People's Republic of China

Full list of author information is available at the end of the article
} 


\section{Background}

Cisplatin is a platinum inorganic complex, extensively used in clinical chemotherapy for many solid tumors. Administration of a high dose of cisplatin is limited due to its nephrotoxic side effects $[1,2]$. Therefore, preventive measures are needed to counteract the renal damage induced by cisplatin. It is now recognized that exosomes/microvesicles as nanoparticles are an integral part of the intercellular microenvironment [3], and may play a pivotal role in cell-to-cell communication [4]. Microvesicles (MVs) are circular fragments of membrane released from the endosomal compartment as exosomes or shed from the surface membranes of most cell types. Exosomes/microvesicles may interact with target cells by surface-expressed ligands and transfer surface receptors, and deliver proteins, mRNA, and bioactive lipids between cells [5, 6]. Recently, many studies have revealed that mesenchymal stem cell (MSC)-derived exosomes or microvesicles can promote tissue regeneration. MVs from human bone marrow-derived MSCs contribute to favor functional and morphological recovery in rodent models of acute kidney injury (AKI) induced by glycerol [7], ischemia/reperfusion [8], and cisplatin [9]. The research implies that exosomes from MSCs may be a novel stem cell-based therapy for kidney diseases. Our previous works indicate that human umbilical cord-derived MSCs (hucMSCs) improve the recovery of ischemia/reperfusion-induced rat renal injury via anti-apoptotic and anti-inflammatory mechanisms [10-12]. We also demonstrated that hucMSC-derived exosomes (hucMSC-Ex) protect against cisplatin-induced renal oxidative stress and apoptosis, alleviate $\mathrm{CCl}_{4}$-induced liver fibrosis, and enhance cutaneous wound healing [13-17]. All the above studies show that hucMSC-Ex can improve the tissue injury. However, it is unknown whether hucMSC-Ex administration before injury can prevent kidney damage in the early stages.

Studies have demonstrated that autophagy is critical for normal proximal tubule function and protection against acute tubular injury $[18,19]$. There is a dynamic feedback between autophagy and cellular energy balance [20]. Autophagy not only plays a principal role in the supply of nutrients for cell survival, but also plays an active role in cellular homeostasis, where it acts as a cytoplasmic quality-control regulator to eliminate longlived or unfolded proteins and damaged organelles $[21,22]$. These reports revealed that the activation of autophagy plays an important role in relieving tissue damage. Whether hucMSC-Ex can promote autophagy activation is unclear.

Herein, we show that hucMSC-Ex had a markedly preventative effect against cisplatin-induced renal toxicity, and we explored the mechanism of action. Our findings indicate that hucMSC-Ex promoted autophagy in renal tubule epithelial cells and kidney tissues by cisplatin induced through the inhibition of mTOR, thus alleviating cell apoptosis and inflammatory response in the early stages.

\section{Methods}

The study was approved by the ethical committee of Jiangsu University (2012258).

\section{Isolation and culture of hucMSCs}

After obtaining parental and ethics committee consent, the fresh umbilical cords were collected and processed within $6 \mathrm{~h}$ as described previously [23]. Umbilical cords were cut into pieces and floated in low glucose Dulbecco's modified Eagle's medium (LG-DMEM) containing 10\% fetal bovine serum (FBS; ExCell Biology, China) and 1\% penicillin and streptomycin. Cord pieces were subsequently incubated at $37{ }^{\circ} \mathrm{C}$ in humid air with $5 \% \mathrm{CO}_{2}$. When well-developed colonies of fibroblast-like cells reached $80 \%$ confluency, cultures were trypsinized into new flasks for further expansion. hucMSCs were identified by fluorescence-activated cell sorting (FACS) and differentiation experiments.

\section{FACS analysis and differentiation studies of hucMSCs}

hucMSCs were resuspended in phosphate-buffered saline (PBS). Cell aliquots $(300 \mu \mathrm{l})$ were incubated with fluorescein isothiocyanate (FITC)-conjugated or phycoerythrin (PE)-conjugated monoclonal antibodies specific for CD13, CD29, CD44, CD90, CD105, HLA-1, CD45, HLA-DR, and CD34 on ice for $30 \mathrm{~min}$. FITC or PE mouse nonimmune isotypic IgG were used as controls. All antibodies were purchased from BD Company. The pluripotency of hucMSCs was confirmed by their ability to differentiate into osteocytes and adipocytes, as described previously [23].

\section{Isolation and characterization of exosomes from hucMSCs and HFL1 cells}

As control cells, human fetal lung fibroblast-1 (HFL1) cells were obtained from Cell Bank, Type Culture Collection Committee, the Chinese Academy of Sciences (Shanghai, China). In order to allow exosome separation, HFL1 cells were cultured with serum-free $\alpha$-modified minimum essential medium ( $\alpha$-MEM); hucMSCs were cultured with serum-free LG-DMEM. The conditioned medium was collected at $48 \mathrm{~h}$. Density gradient centrifugations were executed and the procedure for the extraction and purification of exosomes was performed as described previously $[13,14]$. The purified exosomes were stored at $-86{ }^{\circ} \mathrm{C}$ until use. The expression of the exosomal makers CD81, CD9, and CD63 was detected using Western blot. The purified exosomes were further identified by nanoparticle tracking analysis (NTA) system 
(Version 2.3 Build 0006 BETA2) and transmission electron microscopy.

\section{NTA and transmission electron microscopy}

In order to analyze the characteristics of the exosomes, particle size, substantial shape, and the relative-intensity three-dimensional plot of hucMSC-Ex were tested using the NTA system. The purified hucMSC-Ex were applied to glow-discharged carbon-coated copper grids to detect the precise shape. PTA (phosphor-tungsten acid) staining was conducted for $30 \mathrm{~min}$. The grids were then rinsed with droplets of deionized water and dried. Ultra-thin sections of NRK-52E cells were prepared to observe the formation of autophagic bodies. Transmission electron micrographs were recorded using a Tecnai 12 (Philips, Holland).

\section{In vitro experiment}

Normal rat kidney epithelial (NRK-52E) cells were obtained from Cell Bank (Shanghai, China). NRK-52E cells were pre-incubated with exosomes from hucMSC (200 $\mu \mathrm{g} / \mathrm{ml})$ or exosomes from HFL1 $(200 \mu \mathrm{g} / \mathrm{ml})$ for $30 \mathrm{~min}$, followed by treatment with cisplatin $(8 \mu \mathrm{M})$ for $8 \mathrm{~h}$. 3methyladenine (3MA; $1 \mathrm{mg} / \mathrm{ml}$; Sigma, USA) or rapamycin $(200 \mu \mathrm{g} / \mathrm{ml}$; Sigma) was given for $0.5 \mathrm{~h}$ before exosomes treatment and $1 \mathrm{~h}$ before cisplatin treatment in NRK-52E cells, respectively.

\section{In vivo rat model}

Female Sprague-Dawley rats at 6-8 weeks old, weighing 210-250 g, were used. The animals were kept under standard laboratory conditions $(12 \mathrm{~h}$ light $/ 12 \mathrm{~h}$ dark cycle, and $21 \pm 2{ }^{\circ} \mathrm{C}$ ). Animals were divided into seven groups of six rats each and treated as follows: 1) control group (no cisplatin treatment); 2) PBS group (intraperitoneal injection of a single dose of $5 \mathrm{mg} / \mathrm{kg}$ cisplatin); 3) hucMSC-Ex group $(0.5 \mathrm{~h}$ before cisplatin administration both kidneys in one rat received a renal capsule injection of $200 \mu \mathrm{g}$ hucMSC-Ex); 4) HFL1-Ex group (0.5 h before cisplatin administration both kidneys in one rat received a renal capsule injection of $200 \mu \mathrm{g}$ HFL1-Ex); 5) hucMSC$\mathrm{Ex}+3 \mathrm{MA}$ group $(500 \mu \mathrm{g} / \mathrm{kidney} 3 \mathrm{MA}$ was injected under the kidney capsule before hucMSC-Ex and cisplatin administration); 6) 3MA group (500 $\mu \mathrm{g} /$ kidney 3MA was injected under the kidney capsule before cisplatin administration); and 7) Rapa group (20 $\mu \mathrm{g} /$ kidney 3MA was injected under the kidney capsule before cisplatin administration).

All animals were sacrificed at 3 days after cisplatin injection. Kidney tissue specimens were divided into two. One was quickly excised, rinsed in ice-cold saline, and fixed in $4 \%$ paraformaldehyde, and the paraffinembedded tissues were sliced and stained with hematoxylin and eosin (H\&E). Sections were analyzed and at least 20 random fields were scored by a nephropathologist in a blinded manner. Tubular cell necrosis, tubular dilation, and tubular protein casts $(200 \times$ magnification) in sections were observed and analyzed. Abnormalities were graded by a semiquantitative score from 0 to 4: 0 , no abnormalities; 1 , changes affecting less than $25 \%$ of the sample; 2 , changes affecting 25 to $50 \%$; 3 , changes affecting 50 to $75 \%$; 4 , changes affecting more than $75 \%$. The other specimen was used immediately or frozen at $-86{ }^{\circ} \mathrm{C}$ until further biochemical analysis. Blood samples were collected before and after cisplatin injection $(0,1,2$, and 3 days). Serum creatinine $(\mathrm{Cr})$ and blood urea nitrogen (BUN) levels were measured by an automatic biochemical analyzer (AU2700; Olympus). Serum levels of inflammatory cytokines such as tumor necrosis factor (TNF)- $\alpha$, interleukin (IL)-1 $\beta$ and IL6 were determined by Luminex assays (Luminex 200; Millipore).

\section{Mitochondrial membrane potential assay}

NRK-52E cells seeded in 24-well plates were treated as described for the in vitro experiment. After treatment with cisplatin for $8 \mathrm{~h}$, the cells were fixed with $4 \%$ paraformaldehyde. Mitochondrial membrane potential was measured by a JC- 1 kit according to the manufacturer's protocol (Beyotime, China).

\section{RNA extraction and quantitative real-time PCR}

Total RNA was extracted with Trizol reagent (Invitrogen, USA) from NRK-52E cells and kidney tissues; cDNA was synthesized using a reverse transcription kit according to the manufacturer's instructions (Vazyme, China). Quantitative real-time polymerase chain reaction (qRTPCR) was used to detect the expression of ATG5, ATG7, and $\beta$-actin genes. All samples were examined in triplicate, and all reactions were repeated three times independently using the CFX96 Touch $^{\mathrm{Tm}}$ Real-Time PCR Detection System (Bio-Rad, USA).

\section{TUNEL assay}

Terminal deoxynucleotidyl transferase-mediated dUTP nick end-labeling (TUNEL) staining was used to detect the apoptotic cells. hucMSCs, HFL1 cells, NRK-52E cells, and the kidney tissues were examined using the TdTFragEL DNA fragmentation detection kit according to the manufacturer's protocol (Boster, China). Positive cells were identified as dark brown nuclei under a light microscope (Nikon eclipse TE 3000-U, Japan). The number of apoptotic cells was counted in 20 randomly selected visual fields of blinded samples, at 200× magnification.

\section{Western blot}

Cells, exosomes, and tissues were lysed in RIPA buffer. Protein concentration was determined using the BCA assay kit (Pierce, USA). Sources and dilution factors of primary antibodies were: rabbit polyclonal CD63 (1:1000; 
Bioworld, USA), CD9 (1:1000; Bioworld), CD81 (1:1000; Epitomics, USA), PCNA (1:1000; Bioworld), BCL-XL (1:100; SAB, USA), BCL-2 (1:1000; Bioworld), Bax (1:1000; Bioworld), Cytochrome C (1:500; Abcam, USA), caspase-3 (1:500; Bioworld), IL-1 $\beta$ (1:500; Bioworld,), LC3B (1:500; Abcam), Beclin-1 (1:600; Proteintech, USA), mTOR (1:500; SAB), p-mTOR (1:500; SAB), 4EBP1 (1:200; SAB), p70S6K (1:200; SAB), and mouse monoclonal GAPDH (1:3000; Kang Chen, China). The nucleoprotein and plasma protein was separated by the nuclear and plasma protein isolation kit (Vazyme, China), with the primary antibody NF-kB-P65 in the nucleus (1:500; SAB), primary antibody nucleoprotein Histone (1:1000; SAB). After incubation with the primary antibodies overnight at $4{ }^{\circ} \mathrm{C}$, membranes were washed three times with Tris-buffered saline with $0.05 \%$ Tween-20 and challenged with HRPconjugated goat anti-rabbit or goat anti-mouse antibody (1:2000; Bioworld). Western blot was performed by Luminata $^{\mathrm{TM}}$ crescendo western HRP substrate (Millipore, USA) and analyzed using MD Image Quant Software.

\section{Immunocytochemistry and immunohistochemistry}

NRK-52E cells, treated as described for the in vitro experiment, were fixed in $4 \%$ paraformaldehyde and permeabilized with HEPES-Triton X100 buffer. The paraffin-embedded kidney tissue sections were dewaxed. Endogenous peroxidase activity was then inhibited by exposure to $3 \%$ hydrogen peroxide for $10 \mathrm{~min}$. Subsequently, the sections were boiled for $10 \mathrm{~min}$ in citrate buffer $(\mathrm{pH} 6.0,10 \mathrm{mM})$ for antigen retrieval. The sections were then blocked with 5\% BSA (Boster Bioengineering, Co. Ltd., Wuhan, China) and incubated with TNF- $\alpha$ (1:100; Bioworld) and PCNA (1:100; Bioworld) primary antibody at $37{ }^{\circ} \mathrm{C}$ for $1 \mathrm{~h}$. After the sections were washed with PBS, they were then incubated with diluted secondary antibody for $20 \mathrm{~min}$. Finally, cells were visualized using diaminobenzidine (DAB) substrate and counterstained with hematoxylin for microscopic examination. The number of PCNA-positive cells were counted in 20 randomly selected visual fields of blinded samples, at 200× magnification.

\section{Enzyme-linked immunosorbent assay}

NRK-52E cells were treated as mentioned above. Culture supernatants were collected and cell debris was removed by centrifugation. Supernatants were examined using the rat TNF- $\alpha$ enzyme-linked immunosorbent assay (ELISA) kit according to the manufacturer's protocol (ExCell Biology, China).

\section{Luminex assay}

Female Sprague-Dawley rats were treated as mentioned above. The serum inflammatory cytokines TNF- $\alpha$, IL-1 $\beta$, and IL6 were measured using Luminex kits (Millipore, Billerica, MA, USA) according to the manufacturer's instructions.

\section{Cell transfection and structured illumination microscopy assay}

NRK-52E cells were seeded in 24-well plates and cultured for $24 \mathrm{~h}$, then transfected with mRFP-GFP-LC3 adenovirus according to the manufacturer's protocol (Han Heng Biology, China). Then, cells were cultured on coverslips and divided into four groups: control; PBS; pre-treated hucMSC-Ex; and pre-treated HFL1-Ex. After treatment, the cells were washed with PBS, fixed with $4 \%$ paraformaldehyde, and permeabilized with HEPES-Triton X100 buffer. Finally, the cells were stained with Hoechst33342 for nuclear staining, and the images were acquired with a structured illumination microscopy (Nikon, SIM).

\section{Statistical analysis}

All data from different experiments are expressed as mean \pm SD. Statistical analysis was performed by Student's $t$ test or by analysis of variance (ANOVA) with Newmann-Keuls multicomparison or Dunnett's post hoc tests as appropriate. A two-tailed $P$ value $<0.05$ was considered statistically significant.

\section{Results}

\section{Characterization of hucMSC and hucMSC-Ex}

MSCs isolated from the umbilical cord were characterized by FACS analysis (see Additional file 1: Figure S1A) and induced differentiation (Additional file 1: Figure S1B and C). Exosomes were extracted and purified from hucMSC and were identified by morphology and exosomal markers. The particle size and concentration of exosomes were measured by an NTA system, peaking at $102 \mathrm{~nm}$ diameter (see Additional file 2: Figure S2Aa), a relative-intensity three-dimensional plot (shown in Additional file 2: Figure $\mathrm{S} 2 \mathrm{Ab}$ ), and electron micrograph of phosphotungstanic acid-stained exosomes (Additional file 2: Figure S2Ac). The results of transmission electron microscopy showed that the hucMSC-Ex had a fingerprint-like membrane structure (Additional file 2: Figure S2Ba) and a spheroid shape (Additional file 2: Figure $\mathrm{S} 2 \mathrm{Bb}$ ). The purified hucMSC-Ex expressed exosomal marker proteins such as CD81, CD9, and CD63 (Additional file 2: Figure S2C). TUNEL stain showed that the number of apoptotic cells was not significantly different between normal cultured conditions and serum-free cultured conditions at $48 \mathrm{~h}$ (Additional file 2: Figure S2D). Electron microscope examination showed that hucMSC-Ex expressed CD9 colloidal gold (Additional file 2: Figure S2E).

\section{hucMSC-Ex can prevent cisplatin-induced AKI in vivo}

We determined whether hucMSC-Ex could prevent AKI in rats. Rats were divided into four groups $(n=6$ per group) as follows: 1) control group (rats were treated with PBS intraperitoneally); 2) PBS group (rats were injected with intraperitoneal cisplatin at $5 \mathrm{mg} / \mathrm{kg}$ body 
weight; PBS was injected under the kidney capsule before treatment with cisplatin); 3) hucMSC-Ex group (hucMSC-Ex (200 $\mu \mathrm{g} / \mathrm{kidney)}$ were injected under bilateral renal capsules before treatment with cisplatin); and 4) HFL1-Ex group (HFL1-Ex (exosomes derived from HFL1, as a control for hucMSC-Ex; $200 \mu \mathrm{g} /$ kidney) was injected under the kidney capsule before treatment with cisplatin. All animals were sacrificed at 3 days after cisplatin injection. Representative images of H\&E staining showed that the number of renal tubules with edema and structural damage were significantly reduced in the hucMSC-Ex group (Fig. 1a and b). The number of PCNApositive cells was higher in hucMSC-Ex group compared to the PBS and HFL1-Ex groups (Fig. 1c and d). In contrast, the number of TUNEL-positive cells was lower in the hucMSC-Ex group compared to the PBS and HFL1-Ex groups (Fig. 1e and f). We also measured the serum creatinine $(\mathrm{Cr})$ and blood urea nitrogen (BUN) levels at different times after cisplatin injection. We found that cisplatin led to a sharp increase in both $\mathrm{Cr}$ and BUN levels at 3 days after injection. Treatment with hucMSC-Ex significantly decreased $\mathrm{Cr}$ and BUN levels (Fig. 1g and h). In summary, these results indicate that hucMSC-Ex protects against renal injury induced by cisplatin, but there is no similar preventive action in the HFL1-Ex group.

\section{hucMSC-Ex inhibits cisplatin-induced apoptosis and inflammatory response in vitro}

To determine whether hucMSC-Ex prevents renal tubule epithelial cells from cisplatin-induced apoptosis and secretion of inflammatory cytokines, NRK-52E cells (rat kidney epithelial cells) were treated as described for the in vitro experiment. Mitochondrial membrane potential is considered a sensitive measurement of early apoptosis. Compared with the control group (NRK-52E cells without cisplatin), cisplatin reduced the mitochondrial membrane potential. hucMSC-Ex, but not HFL1-Ex, treatment antagonized the cisplatin-induced decrease in mitochondrial membrane potential (Fig. 2a). We also confirmed the effect of hucMSC-Ex on cisplatin-induced apoptosis using the TUNEL assay. As shown in Fig. $2 \mathrm{~b}$ and c, the number of TUNEL-positive cells, identified as dark brown nuclei, was lower in the hucMSC-Ex group than that in the PBS and HFL1-Ex groups. After treatment with hucMSC-Ex, the expression of apoptosisrelated proteins such as Bax, Cytochrome c, and cleaved Caspase- 3 decreased, but the expression of proliferative and anti-apoptotic proteins such as PCNA, BCL-XL, and BCL-2 increased (Fig. 2d). To determine whether hucMSC-Ex affects the cisplatin-induced inflammatory response in renal tubule epithelial cells, we detected the expression of TNF- $\alpha$ by immunohistochemical staining and IL1- $\beta$ by Western blot. The results showed that the levels of TNF- $\alpha$ (Fig. 2e) and IL1- $\beta$ (Fig. 2f) were reduced in the hucMSC-Ex group compared to that in the PBS group. However, HFL1-Ex showed minimal effects on TNF- $\alpha$ and IL1- $\beta$ expression. Taken together, these results suggest that hucMSC-Ex can inhibit cisplatin-induced apoptosis and secretion of inflammatory cytokines in renal tubule epithelial cells.

\section{hucMSC-Ex activated autophagy in vitro}

Due to autophagy playing significant roles in the homeostasis and physiological function of podocytes in the kidney [24], we detected the effect of hucMSC-Ex on activation of autophagy. NRK-52E cells were pre-treated with hucMSC-Ex or HFL1-Ex and then exposed to cisplatin, and autophagy-related indicators were tested. The results of transmission electron microscopy showed that there were a large number of autophagosomes in hucMSC-Ex-treated cells compared with the PBS and HFL1-Ex groups (Fig. 3a). To better illustrate the autophagy puncta, we used structured illumination microscopy (SIM) which allows us to acquire super-resolution twodimensional images. mRFP-GFP-LC3B NRK-52E cells were pre-treated with hucMSC-Ex or HFL1-Ex and then exposed to cisplatin; SIM images showed that there are a large number of yellow and red dots in hucMSC-Extreated cells compared with the PBS and HFL1-Ex groups (Fig. 3b). hucMSC-Ex also increased the expression of autophagy-related genes including ATG5 and ATG7 by quantitative RT-PCR (see Additional file 3: Figure S3A and $\mathrm{B})(P<0.05)$. The quantitative results of the integrated density of the Western blot image showed that pretreatment with hucMSC-Ex increased the expression of autophagic maker proteins such as LC3B and Beclin-1 (Fig. 3c). The mammalian target of rapamycin (mTOR) signaling pathway plays a critical role in negatively regulating autophagy. We further demonstrated that hucMSC-Ex inhibited the phosphorylation of mTOR and changed the expression of its downstream targets such as 4E-BP1 and P70S6K. The expression of 4E-BP1 was raised, and the expression of P70S6K reduced after hucMSC-Ex administration (Fig. 3d). In summary, these results indicate that pre-treatment with hucMSC-Ex inhibits mTOR phosphorylation and activates autophagy in renal tubule epithelial cells in response to cisplatin.

\section{hucMSC-Ex prevents cisplatin-induced apoptosis and secretion of inflammatory cytokines by activating autophagy in vitro}

Considering the critical role of autophagy in cell apoptosis and tissue inflammation [25, 26], we wanted to know whether the preventative effects of hucMSC-Ex on cisplatin-induced apoptosis and inflammatory response are dependent on autophagy. We treated NRK$52 \mathrm{E}$ cells with hucMSC-Ex in the presence or absence of the autophagic inhibitor 3-methyladenine (3MA). As 


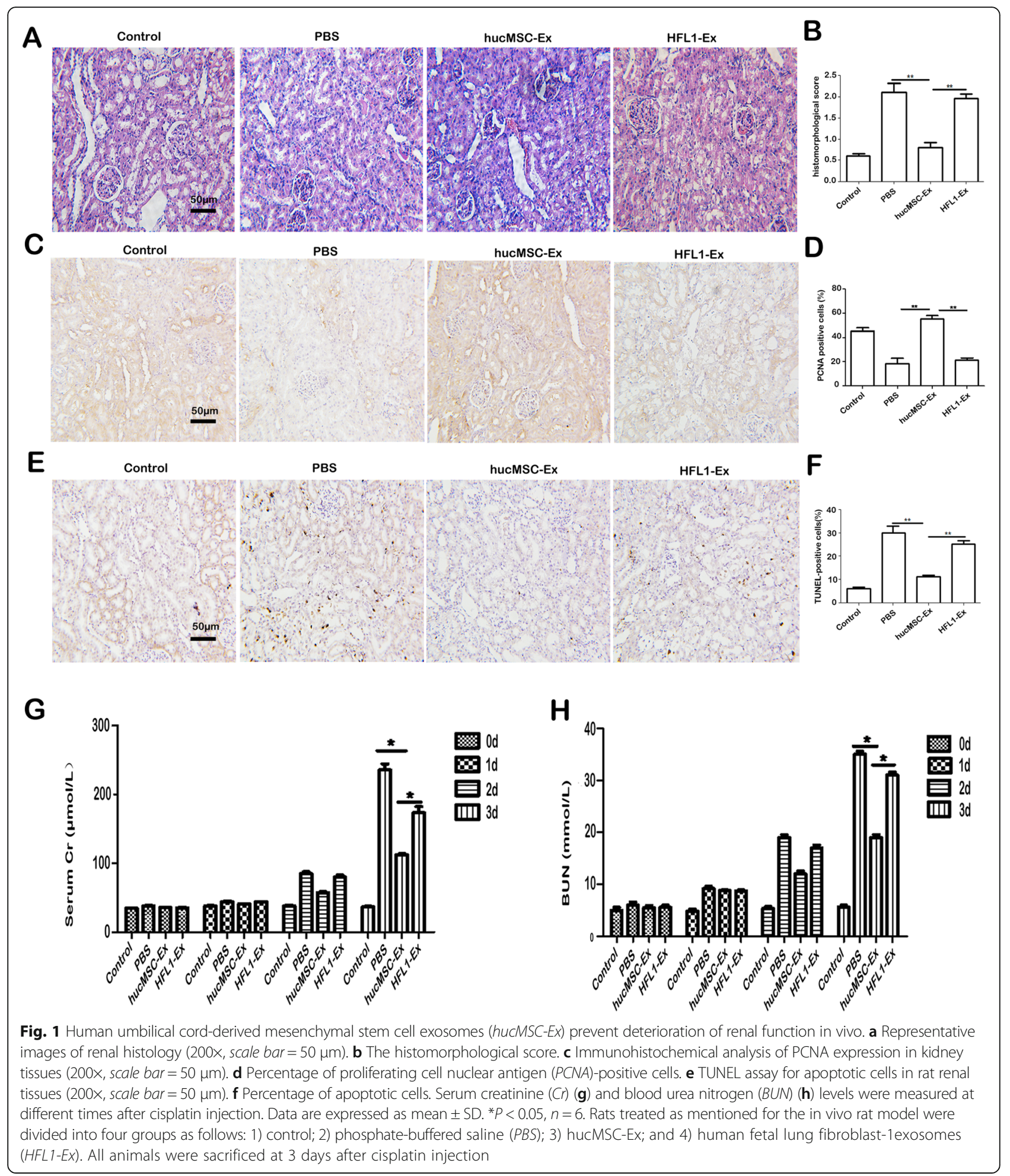

shown in Fig. 4a, 3MA inhibited the hucMSC-Ex-mediated increase of the autophagic marker protein LC3B and antiapoptotic proteins BCL-XL and BCL-2, while it reversed the decrease of the pro-apoptotic protein Bax. However, treatment with the autophagic inducer rapamycin mimicked the effects of hucMSC-Ex, with an increase in LC3B, BCL-2, and $\mathrm{BCL}-\mathrm{XL}$ proteins, and a decrease in Bax protein. We further wanted to know whether the effect of hucMSC-Ex on inflammatory cytokines is also influenced by an autophagic inhibitor. As shown in Fig. 4b, simultaneous treatment 


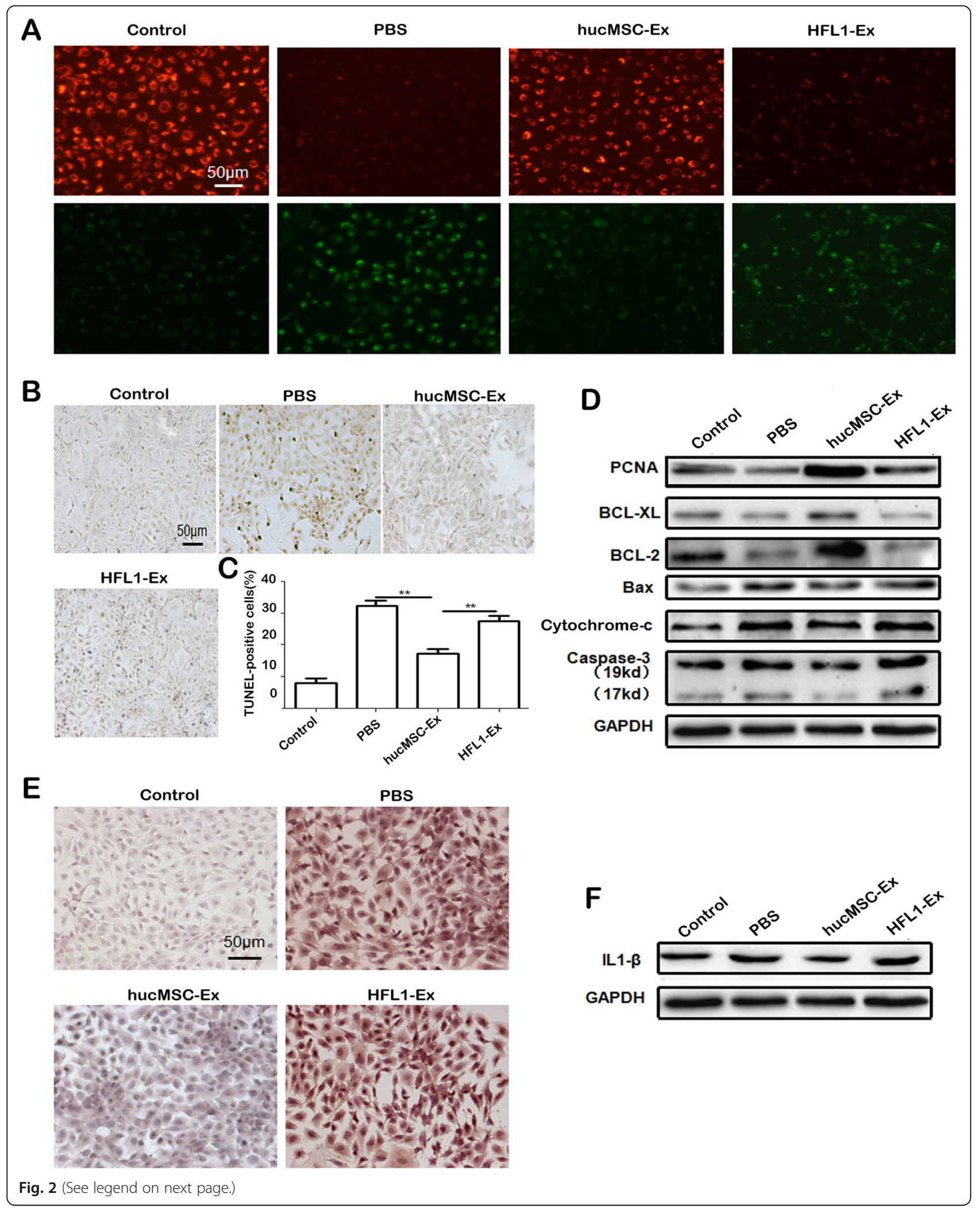




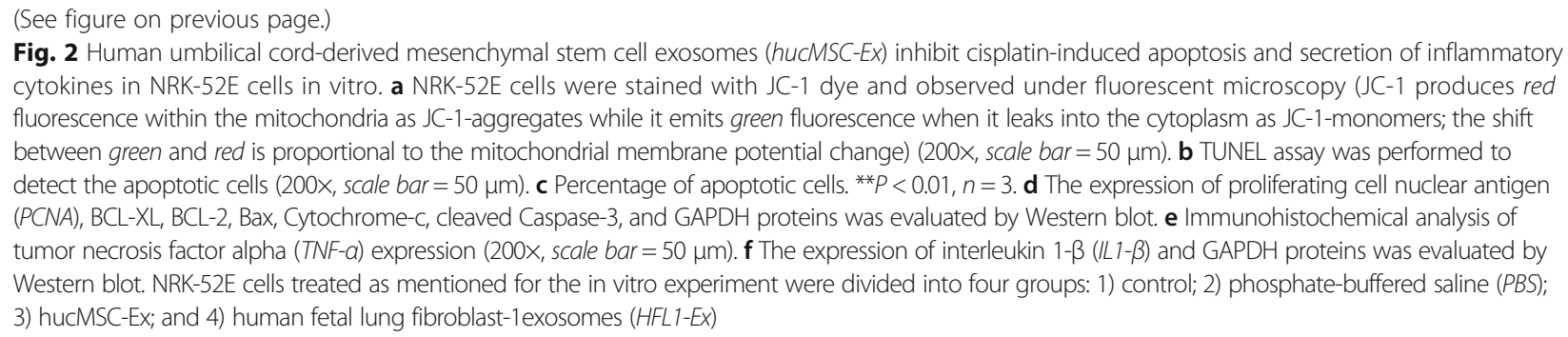

with 3MA abrogated the inhibitory role of hucMSC-Ex in cisplatin-induced IL-1 $\beta$ expression and NF-kB-P65 activation. Treatment with rapamycin reduced IL- $1 \beta$ expression and inactivated NF-kB-P65 to that observed in hucMSCEx-treated cells. The results of ELISA showed that, compared to the PBS group, TNF- $\alpha$ levels decreased in the hucMSC-Ex group but were restored in the hucMSC-Ex + 3MA group (Fig. 4c) $(P<0.05)$. Taken together, these results demonstrated that the activation of autophagy by hucMSCEx may contribute to its preventive effects on cisplatininduced apoptosis and the inflammatory response in vitro.

\section{hucMSC-Ex-mediated activation of autophagy is required for the prevention of cisplatin-induced AKI in vivo}

In order to verify the activation of autophagy induced by the hucMSC-Ex in vivo model, 3MA (500 $\mu \mathrm{g} / \mathrm{kidney)}$ and rapamycin $(20 \mu \mathrm{g} / \mathrm{kidney})$ were respectively injected under the kidney capsule before treatment with cisplatin. Representative images of H\&E staining showed that the number of renal tubules with edema and structural damage were significantly reduced in the hucMSC-Ex group. However, co-injection with 3MA inhibited the protective effect of hucMSC-Ex on the renal structure. Rapamycin had a similar effect in preventing the structural damage of renal tubules to that of hucMSC-Ex (Fig. 5a and b). Furthermore, the number of PCNA-positive cells increased in the hucMSC-Ex group compared to the PBS group. 3MA inhibited the effect of hucMSC-Ex. The expression of PCNA slightly increased in the rapamycin group (Fig. 5c and d). As shown in Fig. 5e and f, the number of TUNEL-positive cells in the hucMSC-Ex and rapamycin groups was reduced, but showed few changes in the hucMSC-Ex + 3MA and HFL1-Ex groups compared to that in the PBS group. The results of immunohistochemical staining showed that TNF- $\alpha$ levels were significantly lower in the hucMSC-Ex and rapamycin groups than in the PBS group. However, TNF- $\alpha$ expression in the hucMSC-Ex + 3MA and 3MA groups showed no significant difference to that in the PBS group (see Additional file 4: Figure S4). Western blotting analyses showed that the expression of the autophagic marker protein LC3B and anti-apoptotic protein BCL-2 increased, while the expression of the pro-apoptotic protein Bax decreased in the hucMSC-Ex group (Fig. 5g). However, the effects of hucMSC-Ex on these proteins were inhibited by 3MA. Luminex assays showed that the serum levels of the inflammatory cytokines TNF- $\alpha$, IL1- $\beta$, and IL6 were significantly lower in the hucMSC-Ex and rapamycin groups than in the PBS group. However, their expression in the hucMSC-Ex + 3MA group showed no significant difference to that in the PBS group (Fig. $5 \mathrm{~h}-\mathrm{j}$ ). Consequently, these results indicate that the activation of autophagy is involved in the preventative role of hucMSCEx in cisplatin-induced AKI.

\section{Discussion}

Increasing evidence suggests that the administration of exogenous mesenchymal stem cells (MSCs) improves the recovery of injured tissue, including acute kidney injury (AKI) [10-12, 27-31]. Several studies have demonstrated that the administration of MSCs could reverse kidney injury through paracrine mechanisms rather than by MSC transdifferentiation [32, 33]. MSC exosomes might be such a paracrine mechanism for cell-to-cell communication. Exosomes are small vesicles released by cells bearing the surface antigens characteristic of the cell of origin [34, 35]. These vesicles may play critical roles in cell communication by transferring RNA, proteins, and bioactive lipids [36]. Exosomes may be more advantageous than stem cells in regenerative medicine due to the avoidance of possible long-term pathologic differentiation of engrafted cells or tumor generation $[37,38]$. The release of exosomes may be constitutive, or a consequence of cell activation by soluble agonists, or physical and chemical stresses such as oxidative stress and hypoxia, or by shear stress [39]. We demonstrated in this study that pre-treatment with hucMSC-Ex could inhibit cisplatin-induced apoptosis and the secretion of inflammatory cytokines in renal proximal tubule epithelial cells. hucMSC-Ex pretreatment suppressed the increase of BUN and serum Cr levels, as well as the deterioration of proximal tubule epithelial cells induced by cisplatin.

The role of autophagy as a degradative pathway is critical in regenerative medicine. Many reports show that basal or physiological autophagy contributes to the maintenance of cellular homeostasis and quality control of proteins and subcellular organelles. Under pathological conditions or cell stress, autophagy is induced which may 


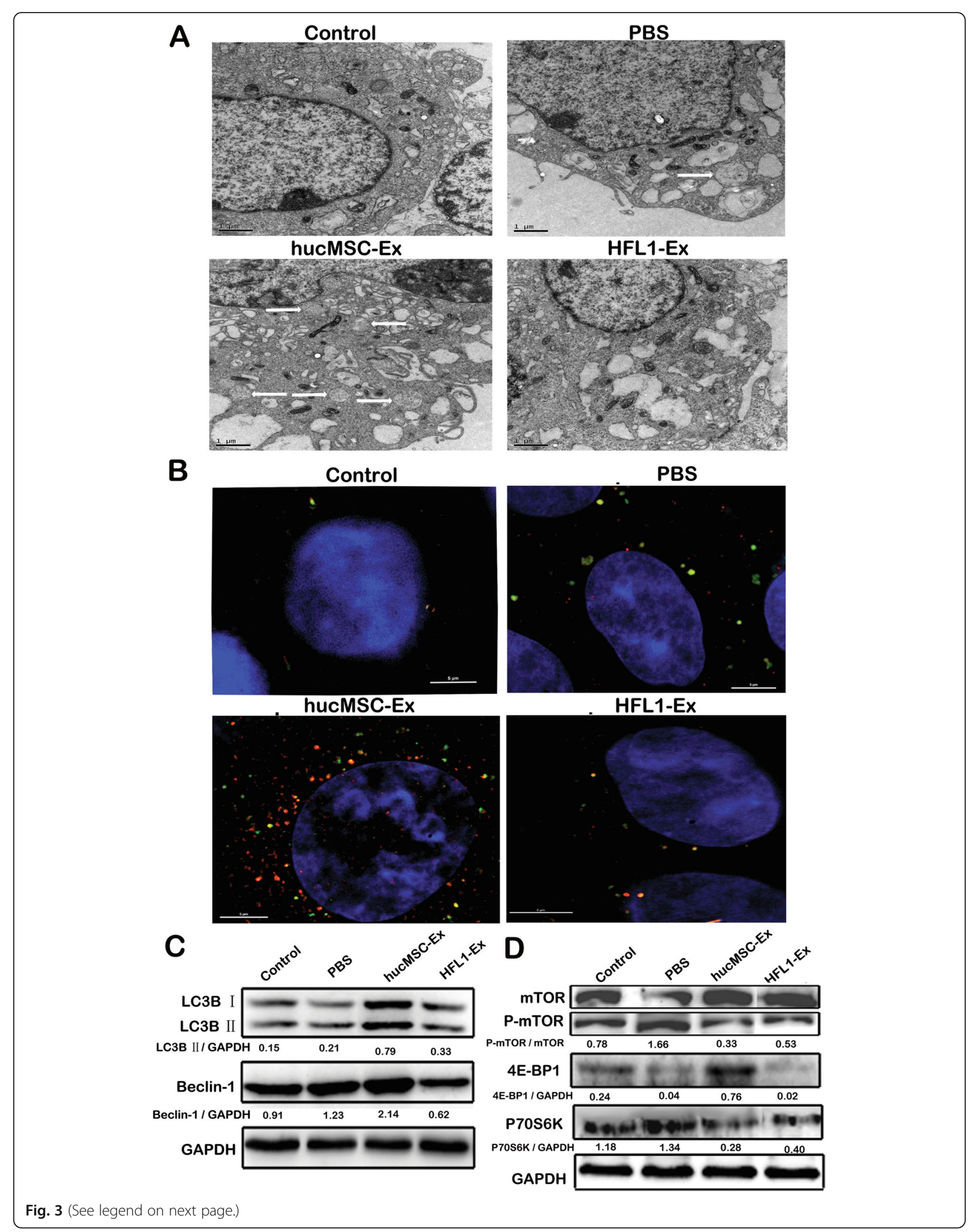


(See figure on previous page.)

Fig. 3 Human umbilical cord-derived mesenchymal stem cell exosomes (huCMSC-Ex) induce autophagy in NRK-52E cells in response to cisplatin. a Transmission electron microscopic examination of autophagosomes in NRK-52E cells. The representative images indicate typical autophagosomes in the cells treated with hucMSC-Ex (scale bar $=1 \mu \mathrm{m}$ ). b SIM examination of autophagosomes in NRK-52E cells. Yellow dots represents autophagosomes, red dots represent autolysosomes (scale bar $=5 \mu \mathrm{m}$ ). Representative images of fluorescent LC3 puncta are shown. $\mathbf{c}$ Western blotting analyses of the expression of the autophagic markers LC-3B and Beclin-1 in NRK-52E cells. $\mathbf{d}$ NRK-52E cells were treated as indicated and the expression of mammalian target of rapamycin (mTOR), phosphorylated mTOR, 4EBP-1, and P70S6K proteins was determined by Western blot. NRK-52E cells treated as mentioned for the in vitro experiment were divided into four groups: 1) control; 2) phosphate-buffered saline (PBS); 3) hucMSC-Ex; and 4) human fetal lung fibroblast-1exosomes (HFL 1-EX)

serve as an adaptive and protective mechanism for cell survival. Autophagy is essential to the homeostasis and physiological function of podocytes in the kidney [40]. Notably, autophagy induction as a self-protection mechanism has been demonstrated in renal tubular cells in experimental models of AKI caused by ischemia-reperfusion and nephrotoxicants such as cisplatin and cyclosporine [41-44]. Recent studies also report that autophagy play an important role in MSC-promoted tissue regeneration $[45,46]$. Baixauli et al. [47] note that the emerging function of exosomes as a means of alleviating intracellular stress conditions in coordination with the autophagy-lysosomal pathway is essential for preserving intracellular protein and RNA homeostasis. However, whether hucMSC-Ex can activate target cell autophagy in advance to prevent tissue injury is not reported. Here, we found that hucMSCderived exosomes can induce target cell autophagy to prevent nephrotoxicity in the early injury stage. In our study, we found that hucMSC-Ex upregulated the expression of the autophagic marker protein LC3B through the inhibition of mTOR phosphorylation. mTOR is an evolutionarily conserved nutrient-sensing serine/threonine protein kinase,

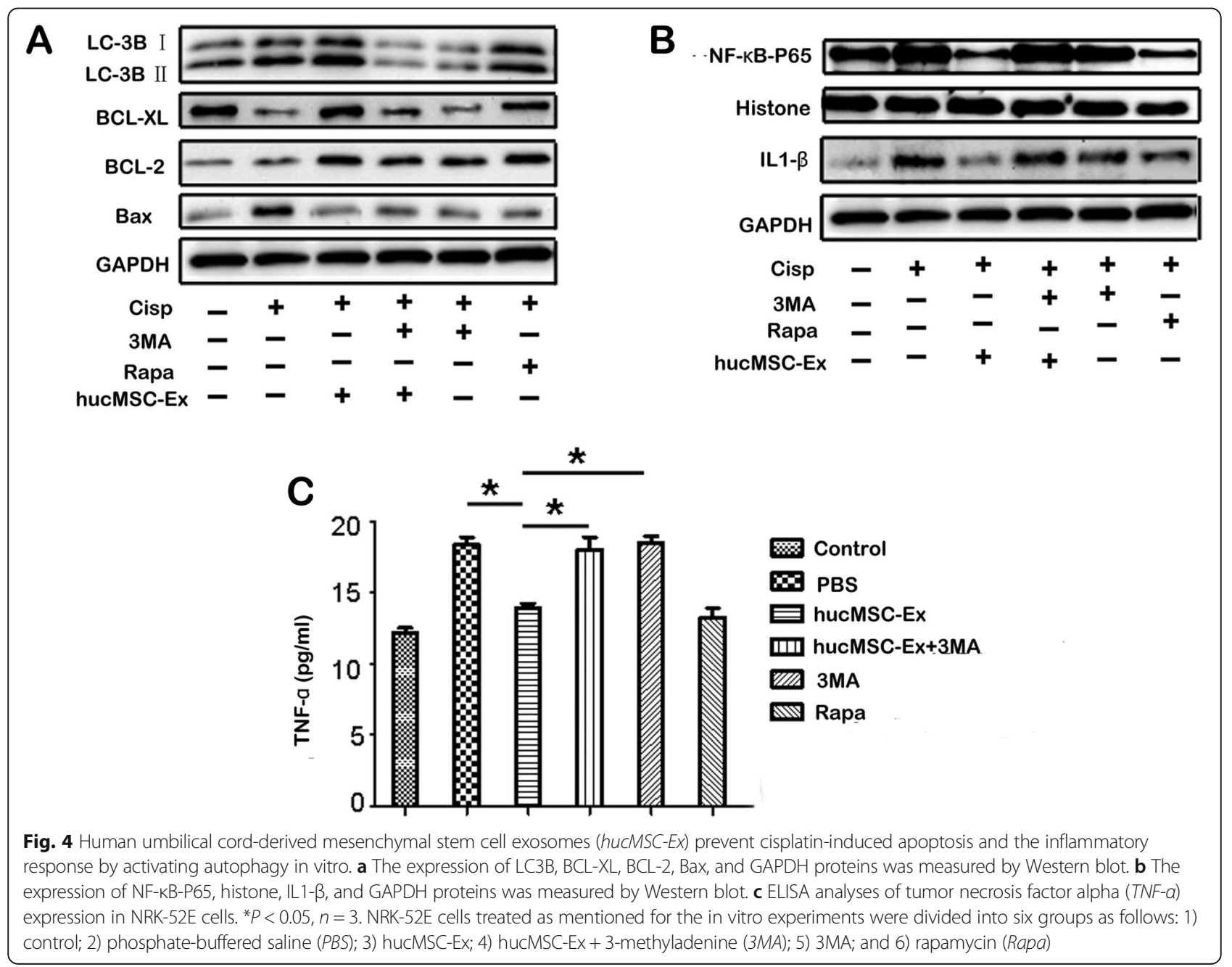




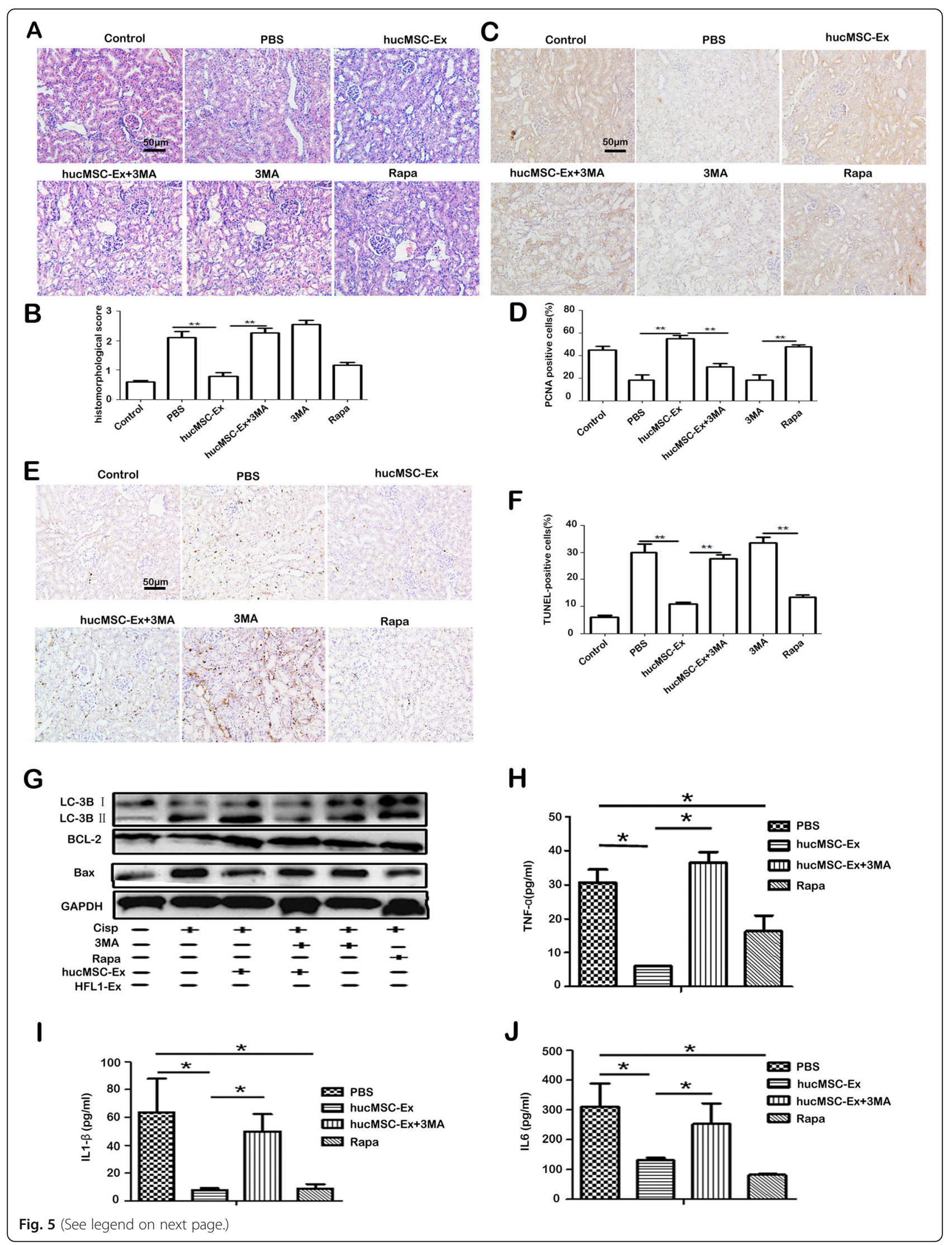


(See figure on previous page.)

Fig. 5 Human umbilical cord-derived mesenchymal stem cell exosomes (huCMSC-Ex) prevent apoptosis and secretion of inflammatory cytokines by activating autophagy in vivo. a Representative images of renal histology (200x, scale bar $=50 \mu \mathrm{m}$ ). $\mathbf{b}$ The histomorphological score. $\mathbf{c}$ Immunohistochemical analysis of PCNA expression in kidney tissues $(200 \times$, scale bar $=50 \mu \mathrm{m})$. $\mathbf{d}$ Percentage of proliferating cell nuclear antigen (PCNA)-positive cells. e TUNEL assay for apoptotic cells in rat renal tissues (200X, scale bar $=50 \mu \mathrm{m})$. f Percentage of apoptotic cells. $\mathbf{g}$ The expression of LC3B, BCL-2, Bax, IL1- $\beta$, and GAPDH proteins in the cisplatin-induced rat model were measured by Western blot. The serum inflammatory cytokines (h) tumor necrosis factor alpha (TNF-a), (i) interleukin-1 $\beta$ (IL1- $\beta$ ), and (j) interleukin-6 (IL6) were measured via Luminex assay. Data are expressed as mean \pm SD. ${ }^{*} P<0.05, n=6$. Rats were divided into six groups as follows: 1) control; 2) phosphate-buffered saline (PBS); 3) hucMSC-Ex; 4) hucMSC-Ex + 3-methyladenine (3MA); 5) 3MA; and 6) rapamycin (Rapa)

with a critical role in regulating protein synthesis and autophagy $[48,49]$. mTOR signaling negatively regulates autophagy, and mTOR suppression by rapamycin contributes to the induction of autophagy [50]. We found that hucMSC-Ex downregulated the expression of phosphorylated mTOR and changed the levels of expression of p70S6K and 4EBP1, suggesting that hucMSC-Ex may activate autophagy by inhibiting the mTOR signaling pathway.

In order to prove whether hucMSC-Ex-mediated autophagy is essential for the inhibition of apoptosis and secretion of inflammatory cytokines, we pre-treated NRK-52E cells with the specific inhibitor of autophagy, 3-methyladenine (3MA). Inhibition of autophagy reduces the effects of hucMSC-Ex on anti-apoptosis and the inhibition of inflammatory cytokines. On the other hand, the autophagic inducer rapamycin has a similar effect on renal protection to that of hucMSC-Ex both in vitro and in vivo, suggesting that induction of autophagy is essential for the protective role of hucMSC-Ex in cisplatin-induced renal injury.

From the above discussion, we propose a model as delineated in Fig. 6. Cisplatin induces apoptosis and inflammation in renal proximal tubule epithelial cells, resulting in acute renal injury. hucMSC-Ex pretreatment activates autophagy through the inhibition of the mTOR signaling pathway in renal proximal tubule epithelial cells, leading

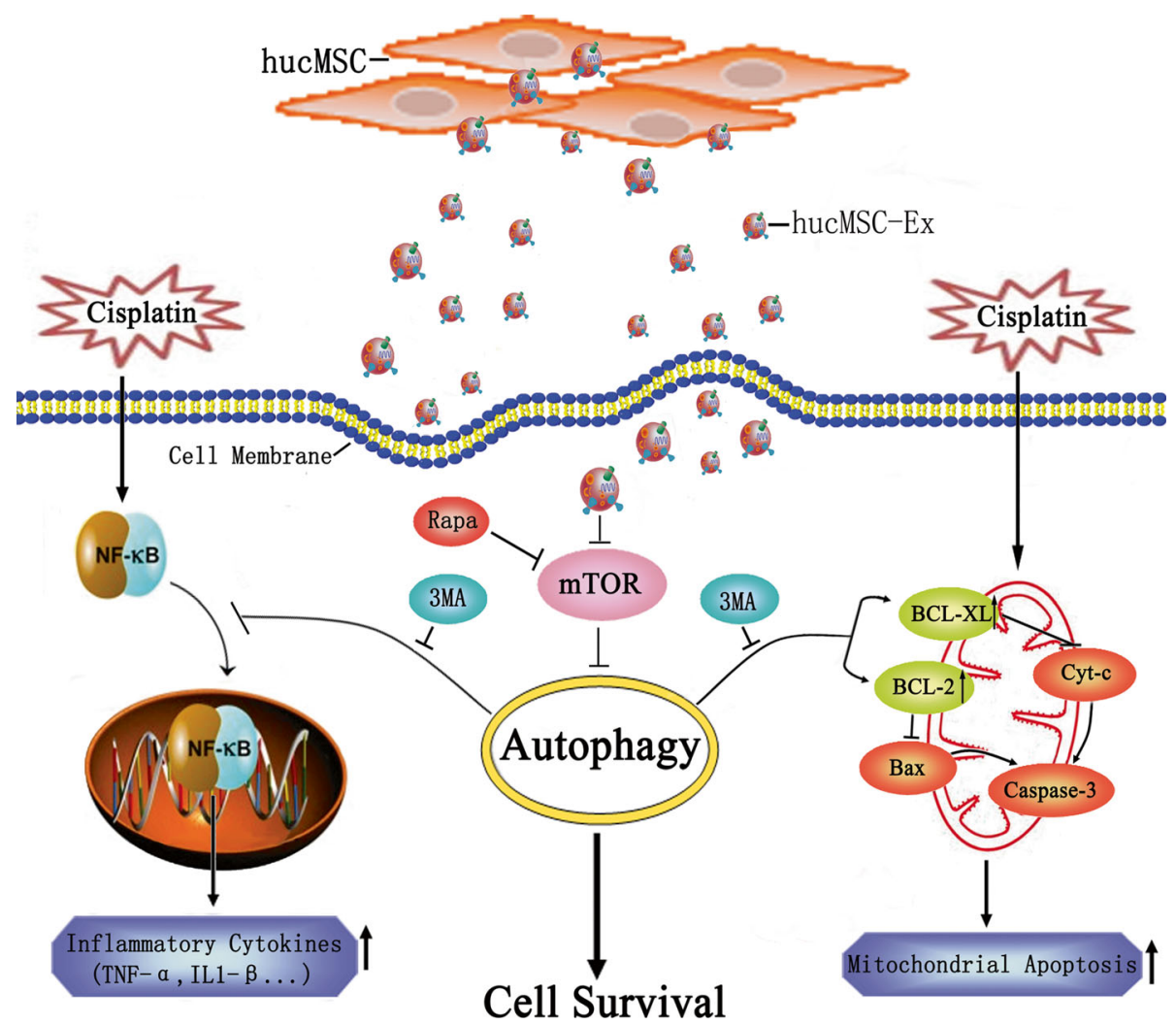

Fig. 6 A proposed model for the mechanism of human umbilical cord-derived mesenchymal stem cell exosome (hucMSC-Ex)-mediated protection against cisplatin-induced AKI. hucMSC-Ex activates autophagy and leads to the reduction in cell apoptosis and inflammatory responses, and the improvement of renal recovery. 3MA 3-methyladenine, IL interleukin, mTOR mammalian target of rapamycin, Rapa rapamycin, TNF tumor necrosis factor 
to the reduction of cell apoptosis and inflammatory responses and the improvement in renal recovery. The preventive effects of hucMSC-Ex on cisplatin-induced AKI can be reversed by the autophagic inhibitor 3-MA. Inactivation of $\mathrm{mTOR}$ by rapamycin mimics the effects of hucMSC-Ex on cisplatin-induced AKI.

\section{Conclusion}

This study reveals that hucMSC-derived exosomes prevent against cisplatin-induced AKI through an autophagyrelated mechanism. These findings provide a basis for the future use of exosomes as a new biological therapeutic approach for renal diseases and injuries.

\section{Additional files}

Additional file 1: Figure S1. Characterization of hucMSCs. (A) Immunophenotyping of hucMSC. Histograms of MSCs stained with antiCD13, CD44, CD29, CD90, CD105, HLA-1, CD45, HLA-DR, and CD34 shown with an overlaid isotype control. (B) Multi-lineage differentiation of hucMSCS. Adipogenic differentiation was assayed by Oil Red O staining (100X, scale bar $=50 \mu \mathrm{m})$. (C) Osteogenic differentiation was assayed by alkaline phosphatase staining $(100 \times$, scale bar $=50 \mu \mathrm{m})$. (PDF $270 \mathrm{~kb})$

Additional file 2: Figure S2. Characterization of hucMSC-derived exosomes. (A) Nanoparticle tracking analysis on characteristics of the particles of hucMSC-Ex: (a) particle size and concentration, (b) the relative-intensity three-dimensional plot, (c) substantial shape of exosomes. (B) The morphology of hucMSC-Ex was observed under transmission electron microscopy (scale bar $=100 \mathrm{~nm}$ ). (C) Western blotting analyses of the expression of exosomal markers CD81, CD9, and CD63 in hucMSC-Ex. (D) TUNEL assay was performed to detect the apoptotic cells (200x, scale bar $=50 \mu \mathrm{m})$. (E) Electron microscope analyses of the expression of exosomal markers CD9 colloidal gold in hucMSC-Ex (scale bar $=500 \mathrm{~nm}$ ). (PDF $228 \mathrm{~kb}$ )

Additional file 3: Figure S3. hucMSC-Ex can activate autophagy in vitro. Quantitative RT-PCR analyses of ATG5 (A) and ATG7 (B) MRNA levels in NRK-52E cells. ${ }^{*} P<0.05, n=3$. (PDF $83 \mathrm{~kb}$ )

Additional file 4: Figure S4. hucMSC-Ex prevents secretion of inflammatory cytokines by activating autophagy in vivo. (A) Immunohistochemical analysis of TNF-a in kidney tissues $(100 \times$, scale bar $=100 \mu \mathrm{m})$. (PDF $197 \mathrm{~kb})$

\section{Abbreviations}

3MA: 3-methyladenine; AKI: Acute kidney injury; BUN: Blood urea nitrogen; Cr: Creatinine; ELISA: Enzyme-linked immunosorbent assay;

FACS: Fluorescence-activated cell sorting; FITC: Fluorescein isothiocyanate; HFL1: Human fetal lung fibroblast-1; hucMSC: Human umbilical cord-derived mesenchymal stem cell; hucMSC-Ex: Human umbilical cord-derived mesenchymal stem cell exosomes; IL: Interleukin; LG-DMEM: Low-glucose Dulbecco's modified Eagle's medium; MEM: Minimum essential medium; MSC: Mesenchymal stem cell; mTOR: Mammalian target of rapamycin; MV: Microvesicle; NTA: Nanoparticle tracking analysis; PBS: Phosphatebuffered saline; PE: Phycoerythrin; GRT-PCR: Quantitative real-time polymerase chain reactiuon; TNF: Tumor necrosis factor; TUNEL: Terminal deoxynucleotidyl transferase-mediated dUTP nick end-labeling

\section{Acknowledgments}

This work was supported by the National Natural Science Foundation of China (grant nos. 81272481, 31140063, and 30840053), the Major Research Plan of Jiangsu Higher Education (grant 15KJA320001), Jiangsu Province "333 Project" fund (grant BRA2015399), Jiangsu Province for Outstanding Sci-Tech Innovation Team in Colleges and Universities (grant SJK2013-10), the opening project of the Key Laboratory of Embryo Molecular Biology, Ministry of Health of China, and Shanghai Key Laboratory of Embryo and Reproduction Engineering (grant
KF201601), and Project Funded by the Priority Academic Program Development of Jiangsu Higher Education Institutions.

\section{Funding}

The National Natural Science Foundation of China (81272481); the National Natural Science Foundation of China (31140063); and the National Natural Science Foundation of China (30840053).

Availability of data and materials

Not applicable.

\section{Authors' contributions}

BW: conception and design, collection and/or assembly of data, data analysis and interpretation, and manuscript writing. HJ: conception and design, collection and assembly of data, data analysis and interpretation, and manuscript writing. BZ: collection and/or assembly of data and data analysis, and manuscript writing. JW: collection and/or assembly of data and data analysis. CJ: data analysis and interpretation. XZ: provision of study material and interpretation. YY: data analysis and interpretation. LY: collection and/or assembly of data. JY: data analysis and interpretation. HQ: study design, data analysis and interpretation, manuscript writing, and final approval of manuscript. WX: study design, data analysis and interpretation. All authors read and approved the final manuscript.

\section{Competing interests}

The authors declare that they have no competing interests.

\section{Consent for publication}

Not applicable.

\section{Ethical approval and consent to participate}

The study was approved by the ethical committee of Jiangsu University (2012258)

\section{Author details}

'Key Laboratory of Laboratory Medicine of Jiangsu Province, School of Medicine, Jiangsu University, 301 Xuefu Road, Zhenjiang, Jiangsu 212013, People's Republic of China. ${ }^{2}$ The Second Affiliated Hospital of Soochow University, Suzhou, Jiangsu, People's Republic of China.

Received: 4 August 2016 Revised: 5 December 2016 Accepted: 20 December 2016 Published online: 08 April 2017

\section{References}

1. Arany I, Safirstein RL. Cisplatin nephrotoxicity. Semin Nephrol. 2003;23:460-4.

2. Stewart JD, Bolt HM. Cisplatin-induced nephrotoxicity. Arch Toxicol. 2012:86:1155-6

3. Deng ZB, Zhuang X, Ju S, Xiang X, Mu J, Liu Y, et al. Exosome-like nanoparticles from intestinal mucosal cells carry prostaglandin E2 and suppress activation of liver NKT cells. J Immunol. 2013;190:3579-89.

4. Van Niel G, Raposo G, Candalh C, Boussac M, Hershberg R, Cerf-Bensussan $\mathrm{N}$, et al. Intestinal epithelial cells secrete exosome-like vesicles. Gastroenterology. 2001;121:337-49.

5. Valadi H, Ekström K, Bossios A, Sjöstrand M, Lee JJ, Lötvall JO. Exosomemediated transfer of mRNAs and microRNAs is a novel mechanism of genetic exchange between cells. Nat Cell Biol. 2007;9:654-9.

6. Kim HS, Choi DY, Yun SJ, Choi SM, Kang JW, Jung JW, et al. Proteomic analysis of microvesicles derived from human mesenchymal stem cells. J Proteome Res. 2012;11:839-49.

7. Bruno S, Grange C, Deregibus MC, Calogero RA, Saviozzi S, Collino F, et al. Mesenchymal stem cell-derived microvesicles protect against acute tubular injury. J Am Soc Nephrol. 2009;20:1053-67.

8. Gatti S, Bruno S, Deregibus MC, Sordi A, Cantaluppi V, Tetta C, et al. Microvesicles derived from human adult mesenchymal stem cells protect against ischemia-reperfusion induced acute and chronic kidney injury Nephrol Dial Transplant. 2011;26:1474-83.

9. Bruno S, Grange C, Collino F, Deregibus MC, Cantaluppi V, Biancone L, et al. Microvesicles derived from mesenchymal stem cells enhance survival in a lethal model of acute kidney injury. PLoS One. 2012;7:e33115. 
10. Cao H, Qian H, Xu W, Zhu W, Zhang X, Chen Y, et al. Mesenchymal stem cells derived from human umbilical cord ameliorate ischemia/reperfusioninduced acute renal failure in rats. Biotechnol Lett. 2010;32:725-32.

11. Chen Y, Qian H, Zhu W, Zhang X, Yan Y, Ye S, et al. Hepatocyte growth factor modification promotes the amelioration effects of human umbilical cord mesenchymal stem cells on rat acute kidney injury. Stem Cells Dev. 2011;20:103-13.

12. Peng $X, X u H$, Zhou $Y$, Wang B, Yan $Y$, Zhang $X$, et al. Human umbilical cord mesenchymal stem cells attenuate cisplatin-induced acute and chronic renal injury. Exp Biol Med. 2013;238:960-70.

13. Zhou Y, Xu H, Xu W, Wang B, Wu H, Tao Y, et al. Exosomes released by human umbilical cord mesenchymal stem cells protect against cisplatininduced renal oxidative stress and apoptosis in vivo and in vitro. Stem Cell Res Ther. 2013;4(2):34.

14. Li T, Yan Y, Wang B, Qian H, Zhang X, Shen L, et al. Exosomes derived from human umbilical cord mesenchymal stem cells alleviate liver fibrosis. Stem Cells Dev. 2013;22:845-54.

15. Zhang B, Wang M, Gong A, Zhang X, Wu X, Zhu Y, et al. HucMSC-exosome mediated Wnt4 signaling is required for cutaneous wound healing. Stem Cells. 2015;33:2158-68.

16. Zhang B, Wu X, Zhang $X$, Sun $Y$, Yan $Y$, Shi H, et al. Human umbilical cord mesenchymal stem cell exosomes enhance angiogenesis through the Wnt4/ß-catenin pathway. Stem Cells Transl Med. 2015:4:513-22.

17. Zhang B, Shi Y, Gong A, Pan Z, Shi H, Yang H, et al. HucMSC exosomedelivered $14-3-3 \zeta$ orchestrates self-control of the Wnt response via modulation of YAP during cutaneous regeneration. Stem Cells. 2016;34(10):2485-500

18. Isaka Y, Kimura T, Takabatake $Y$. The protective role of autophagy against aging and acute ischemic injury in kidney proximal tubular cells. Autophagy. 2011;7:1085-7.

19. Kimura T, Takabatake Y, Takahashi A, Kaimori JY, Matsui I, Namba T, et al. Autophagy protects the proximal tubule from degeneration and acute ischemic injury. J Am Soc Nephrol. 2011;22:902-13.

20. Singh R, Cuervo AM. Autophagy in the cellular energetic balance. Cell Metab. 2011;13:495-504.

21. Yorimitsu T, Klionsky DJ. Autophagy: molecular machinery for self-eating Cell Death Differ. 2005;2:1542-52.

22. Levine B, Kroemer G. Autophagy in the pathogenesis of disease. Cell. 2008;132:27-42

23. Qiao C, Xu W, Zhu W, Hu J, Qian H, Yin Q, et al. Human mesenchymal stem cells isolated from the umbilical cord. Cell Biol Int. 2008;32:8-15.

24. Chien CT, Shyue SK, Lai MK. BCl-xL augmentation potentially reduces ischemia/reperfusion induced proximal and distal tubular apoptosis and autophagy. Transplantation. 2007;84:1183-90.

25. Alayev A, Berger SM, Kramer MY, Schwartz NS, Holz MK. The combination of rapamycin and resveratrol blocks autophagy and induces apoptosis in breast cancer cells. J Cell Biochem. 2015;116:450-7.

26. Wen Z, Fan L, Li Y, Zou Z, Scott MJ, Xiao G, et al. Neutrophils counteract autophagy-mediated anti-inflammatory mechanisms in alveolar macrophage: role in posthemorrhagic shock acute lung inflammation. J Immunol. 2014;193:4623-33.

27. Eliopoulos N, Zhao J, Bouchentouf M, Forner K, Birman E, Yuan S, et al. Human marrow-derived mesenchymal cells decrease cisplatin renotoxicity in vitro and in vivo and enhance survival of mice post-intraperitoneal injection. Am J Physiol Renal Physiol. 2010;299:1288-98.

28. Morigi M, Rota C, Montemurro T, Montelatici E, Lo Cicero V, Imberti B, et al. Life-sparing effect of human cord blood-mesenchymal stem cells in experimental acute kidney injury. Stem Cells. 2010;28:513-22.

29. Morigi M, Introna M, Imberti B, Corna D, Abbate M, Rota C, et al. Human bone marrow-mesenchymal stem cells accelerate recovery of acute renal injury and prolong survival in mice. Stem Cells. 2008;26:2075-82.

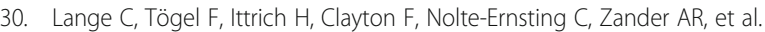
Administered mesenchymal stem cells enhance recovery from ischemia/ reperfusion-induced acute renal failure in rats. Kidney Int. 2005;68:1613-7.

31. Humphreys BD, Bonventre JV. Mesenchymal stem cells in acute kidney injury. Annu Rev Med. 2008;59:311-25.

32. Zarjou A, Kim J, Traylor AM, Sanders PW, Balla J, Agarwal A, et al. Paracrine effects of mesenchymal stem cells in cisplatin-induced renal injury require heme oxygenase-1. Am J Physiol Renal Physiol. 2011;300:254-62.
33. He J, Wang $Y$, Sun S, Yu M, Wang C, Pei X, et al. Bone marrow stem cellderived microvesicles protect against renal injury in the mouse remnant kidney model. Nephrology. 2012;17:493-500.

34. Schorey JS, Bhatnagar S. Exosome function: from tumor immunology to pathogen biology. Traffic. 2008;9:871-81.

35. Mause SF, Weber C. Microparticles: protagonist of a novel communication network for intercellular information exchange. Circ Res. 2010;107:1047-57.

36. Morel O, Toti F, Hugel B, Freyssinet JM. Cellular microparticles: a disseminated storage pool of bioactive vascular effectors. Curr Opin Hematol. 2004;11:156-64.

37. Lai RC, Chen TS, Lim SK. Mesenchymal stem cell exosome: a novel stem cell-based therapy for cardiovascular disease. Regen Med. 2011;6(4):481-92.

38. Baglio SR, Pegtel DM, Baldini N. Mesenchymal stem cell secreted vesicles provide novel opportunities in (stem) cell-free therapy. Front Physiol. 2012;3:359.

39. Ratajczak J, Wysoczynski M, Hayek F, Janowska-Wieczorek A, Ratajczak MZ. Membrane-derived microvesicles: important and underappreciated mediators of cell-to-cell communication. Leukemia. 2006;20:1487-95.

40. Bray K, Mathew R, Lau A, Kamphorst JJ, Fan J, Chen J, et al. Autophagy suppresses RIP kinase-dependent necrosis enabling survival to mTOR inhibition. PLoS One. 2012;7:e41831.

41. Suzuki C, Isaka Y, Takabatake Y, Tanaka H, Koike M, Shibata M, et al. Participation of autophagy in renal ischemia/reperfusion injury. Biochem Biophys Res Commun. 2008;368:100-6.

42. Inoue K, Kuwana H, Shimamura Y, Ogata K, Taniguchi Y, Kagawa T, et al. Cisplatin-induced macroautophagy occurs prior to apoptosis in proximal tubules in vivo. Clin Exp Nephrol. 2010;14:112-22.

43. Jiang M, Liu K, Luo J, Dong Z. Autophagy is a renoprotective mechanism during in vitro hypoxia and in vivo ischemia-reperfusion injury. Am J Pathol. 2010;176:1181-92.

44. Periyasamy-Thandavan S, Jiang M, Wei Q, Smith R, Yin XM, Dong Z. Autophagy is cytoprotective during cisplatin injury of renal proximal tubular cells. Kidney Int. 2008;74:631-40.

45. Dang $\mathrm{S}, \mathrm{Xu}$ H, Xu C, Cai W, Li Q, Cheng Y, et al. Autophagy regulates the therapeutic potential of mesenchymal stem cells in experimental autoimmune encephalomyelitis. Autophagy. 2014;10:1301-15.

46. Shin JY, Park HJ, Kim HN, Oh SH, Bae JS, Ha HJ, et al. Mesenchymal stem cells enhance autophagy and increase $\beta$-amyloid clearance in Alzheimer disease models. Autophagy. 2014;10:32-44.

47. Baixauli F, López-Otín C, Mittelbrunn M. Exosomes and autophagy: coordinated mechanisms for the maintenance of cellular fitness. Front Immunol. 2014;5:403.

48. Mizushima N. The role of the Atg1/ULK1 complex in autophagy regulation. Curr Opin Cell Biol. 2010;22:132-9.

49. Ravikumar B, Sarkar S, Davies JE, Futter M, Garcia-Arencibia M, GreenThompson ZW, et al. Regulation of mammalian autophagy in physiology and pathophysiology. Physiol Rev. 2010;90:1383-435.

50. Yang J, Zhao X, Patel A, Potru R, Azizi-Ghannad S, Dolinger M, et al. Rapamycin inhibition of $m T O R$ reduces levels of the $\mathrm{Na}+/ \mathrm{H}+$ exchanger 3 in intestines of mice and humans, leading to diarrhea. Gastroenterology. 2015;149:151-62.

\section{Submit your next manuscript to BioMed Central and we will help you at every step:}

- We accept pre-submission inquiries

- Our selector tool helps you to find the most relevant journal

- We provide round the clock customer support

- Convenient online submission

- Thorough peer review

- Inclusion in PubMed and all major indexing services

- Maximum visibility for your research

Submit your manuscript at www.biomedcentral.com/submit 